\title{
From Electrojet to ITER: India's Journey in Experimental Plasma Physics
}

\author{
P. I. John \\ 7C, Skyline Hillview, Kanjikuzhy, Kottayam, 686004, India
}

Copyright $\bigcirc 2017$ by authors, all rights reserved. Authors agree that this article remains permanently open access under the terms of the Creative Commons Attribution License 4.0 International License

\begin{abstract}
India has an international presence in Plasma Physics and its diverse applications such as thermonuclear fusion, material processing, strategic and environmental applications and plasma devices. From a modest start in the early 1970 s, we have made great strides in the field of experimental plasma physics. Capacity building in techniques relevant to plasma production, manipulation and parameter control, pulsed power, creation of magnetic fields of complex geometries, clean vacuum and pumping systems, development and deployment of diagnostics to enable understanding of fundamental processes in plasmas and computer simulation to model plasma phenomena have been truly remarkable. Parallel to this, a community of physicists, engineers and computer experts has grown and matured. Funding mechanisms and financial support essential to broad base the research and development activity by drawing in Universities and education institutes have been nucleated. It is through these activities that the human resource and technology development essential to sustain India's ambitious forays into magnetic confinement fusion and industrial and strategic plasma applications has taken place. This paper is an attempt to give a historical perspective to this journey, which started at the Physical Research Laboratory, Ahmedabad and later, involved the Institute for Plasma Research at Gandhinagar, many DAE Institutions, IITs and Universities.
\end{abstract}

Keywords Plasma Physics, Magnetic Confinement Fusion, Industrial Plasma, Intense Electron Beam, Toroidal Plasma, Tokamak, National Fusion Programme, ITER

\section{Introduction}

India has an international presence in Plasma Physics and its diverse applications such as thermonuclear fusion, material processing and plasma devices. It is one of the seven partners building ITER, which is expected to prove fusion's technical viability as an energy source. Research in Plasma
Physics is being pursued in national centres like the Institute for Plasma Research-Gandhinagar, Bhabha Atomic Research Centre-Mumbai, Centre for Plasma Physics-Guwahati and Saha Institute for Nuclear Physics-Kolkata and in IITs and Universities. It has active international collaboration with European Union and US. National and international conferences in plasma physics and allied fields are held regularly. A large number of students are trained in both fundamental and applied plasma physics and in both theory and experiments.

In this presentation, I shall attempt an overview of the development of experimental plasma physics as it happened first at the Physical Research Laboratory and later at the Institute for Plasma Research. Historical surveys often simplify events and distort time. The purpose is to convey a sense of how activities evolved the struggles and occasional triumphs.

Plasma Physics in India began in Allahabad in 1920 with Saha's fundamental theoretical investigations of ionization rates in thermal equilibrium plasmas [1]. In 1938, Saha moved to the University of Calcutta to establish the Institute for Nuclear Physics. With setting up of the first cyclotron, experiments with the duoplasmatron ion source started. A toroidal pinch experiment had operated at the Tata Institute of Fundamental Research in the early 1960s. With the failure of the ZETA experiment in Harwell in England, this work was stopped. The practitioners dispersed; some to join the new accelerator programme of the Department of Atomic Energy and others to set up the Society for Applied Microwave Electronics Engineering and Research (SAMEER).

Vikram Sarabhai picked up the threads again when he assembled a group in PRL in the early 1970s. A theory effort took shape in the backdrop of space science research at PRL with the infusion of personnel from TIFR, IIT Delhi and US universities. The group also acquired experimental expertise with the induction of personnel from Aligarh Muslim University and from the cosmic ray and solar radio burst groups at PRL. The plan was to establish an experimental programme in basic plasma physics with strong orientation 
towards the simulation of space plasma phenomena. However, there was an unstated purpose of eventually acquiring the skills necessary for fusion research. The group transformed itself into the Plasma Physics Programme funded by DST in 1982, which in 1984 became the Institute for Plasma Research.

\section{Simulation of Electrojet Instabilities}

Equatorial electrojet is an electric current carried by electrons across the magnetic field in the $\mathrm{E}$ region of the Earth's ionosphere. Rocket experiments from PRL had observed instabilities in the electrojet region. The first experiment was designed to simulate ionospheric conditions in which cross-field electron streaming drives collisional plasma instabilities. A device with two co-axial annular plasma columns using RF discharge in an axial magnetic field was set up. The radial density gradient could be controlled by the relative densities in the axial columns. The radial electric field was imposed by line charging using biased end plates. The azimuthal ExB drift of electrons would drive both low frequency and high frequency instabilities. Azimuthally separated Langmuir probes detected instabilities and measured the phase velocity and power spectrum.

High frequency $(\omega>v i>\Omega i)$ instabilities are observed when electrons stream in the $\mathrm{E} \times \mathrm{B}$ direction past ions with a velocity exceeding the ion acoustic speed. The observed phase velocities and frequencies agree with the results of the Farley-Buneman instability theory, modified to take account of finite $\mathrm{k} \|$ [2]. The measured instability spectrum for potential fluctuations goes as $\mathrm{k}-\mathrm{n}$ with $\mathrm{n}=2-3.5$. The low frequency cross field instability [3] was investigated for different values of externally applied radial electric field. The dispersion relation obtained experimentally differs significantly from predictions of linear theory for strong electric fields. K-spectra for density and potential fluctuations follow power law with indices $(-3 \cdot 7 \pm 0 \cdot 5)$ and $(-5 \cdot 6 \pm 0.6)$ respectively. At large values of applied electric field, the main cross field modes appear to give rise to a number of other modes possibly through non-linear wave-wave interactions. Large amplitude $\mathrm{m}=2$ mode displays strong sidebands indicating particle trapping by the non-linear waves. Non- linear wave-wave and wave particle interactions, thus, appear to play at important role in the saturation of the cross field instability [4].

\section{Single Particle Confinement}

With the success of the first experiment, a more sophisticated experiment on the confinement of single particles in a non-adiabatic magnetic mirror was attempted. The motivation was theoretical work done at PRL, which attributed the non-adiabatic loss of particles from a mirror trap to tunneling from the adiabatic potential well by particles of energy lower than the maximum height of the potential barrier and predicted the decay of the number of particles from the trap with multiple life times. An ultra-high vacuum chamber with multiple water cooled solenoids forming a symmetric mirror was set up. Electrons were injected into this from a thermionic injector. An electrode beyond a mirror throat collected electrons leaving the trap. The low electron density of the order of $104 / \mathrm{cc}$ ruled out collective behaviour. The experimental results conformed to theoretical predictions in some essential aspects; namely, the existence of more than one decay time, their dependence on the magnetic field, its gradient and the particle energy [5-6]. The ratio of the values of the slope of life time versus magnetic field at different pitch angles, radial positions and particle densities agreed with theoretical predictions.

\section{Ion Acoustic Solitons}

Interest in ion acoustic waves was triggered by a visit by Igor Alexeff from the University of Tennessee. A large quiescent plasma device with filament produced plasma was set up and led to a number of experiments on soliton propagation in density gradients, its modification by ponderomotive force produced by RF waves, reflection of solitons in sharp density gradients etc. [7-9]. The novel observation of rarefaction solitons and their fissioning while propagating away from the launcher was a highlight of this phase. After conversion of the device into a double plasma device, experiments on the formation of ion acoustic double layers resembling asymmetric ion-holes were pursued. When electrons are injected into the target plasma, modulation of the beam through step potential leads to excitation of ion-acoustic fluctuation. The ion-acoustic fluctuation, growing away from the grids separating source and target plasmas, developed into weak asymmetric ion-acoustic double layer. Other experiments done in this device included studies on the evolution of ion matrix sheaths driven by high negative voltage pulses [10-11].

\section{Critical Velocity Phenomenon}

Alfven had proposed that when a plasma and neutral gas in relative motion across a magnetic field interact, rapid ionization of the neutral gas would happen when the velocity exceed a critical value $\mathrm{Vcr}=[2 \mathrm{eVi} / \mathrm{Mi}] 1 / 2$ where $\mathrm{Mn}$ is the atomic mass of the neutral gas and $\mathrm{Vi}$ is the ionization potential. A theory developed at PRL had proposed the existence of a threshold velocity determined by the kinetic energy of the ion species for the interaction to happen. The experimental device produced fast moving plasma streams from a coaxial plasma gun which impinged on a neutral gas cloud formed by the release of gas into vacuum through a fast opening gas valve. The experiment confirmed the critical 
velocity phenomenon, while showing the absence of the threshold velocity [12-14].

\section{Intense Electron Beam-Plasma Interaction}

Even at moderate beam energies and currents, say few hundred $\mathrm{keV}$ and tens of $\mathrm{kA}$, electron beams have interesting physical properties. The associated space charge is intense, producing self-electric fields of the order of $\mathrm{MV} / \mathrm{cm}$. The self-magnetic fields are strong enough to turn the beam trajectories into complex shapes or even reflex the beam electrons. Injected into the plasma, the electric fields are cancelled by the expulsion of plasma electrons. The rising front of the self-magnetic field drives return currents. When such beams are propagated in plasmas, very interesting effects involving the interaction of beam fields with plasmas would happen.

The basic elements of sub-microsecond pulse power technology are high energy density capacitors, high pressure spark gaps, Marx generators and transmission lines for pulse formation, high voltage switches etc. Work in this field had started in the early 1960's at Aldermaston and we had to catch up with more than a decade's accumulated knowledge. A very important lesson learned was that although conceptually simple, the complexity lies in practice and prescriptions. For example, the water-vacuum interface was a Perspex flange on which the cathode is attached. This is a classic triple junction where dielectric, metal and vacuum meets and is prone to radial flashover. We had to understand how to divert the radial electrical field away from the dielectric to stop the avalanche build up along the dielectric surface.

A 20 stage Marx bank using Maxwell capacitors was housed in an oil-filled tank charged through a water charging line and discharged through a spark gap array. A dielectric surface flash triggered the first gap and the inter-capacitor coupling optimized by the layout ensured reliable Marx erection. The Marx output was used to charge a $100 \mathrm{nS}$ water pulse forming line which switches through an overvolted water switch into a graphite cathode diode generating a 300 $\mathrm{kV} 30 \mathrm{kA} 100$ nanosecond annular beam. This is nominally a beam carrying power in Gigawatts [15].

By the time we were ready with the beam, laminar beam plasma interaction studies had been exhausted and we decided to get into the novel territory of rotating electron beams. When a paraxial beam travels through a non-adiabatic cusp magnetic field, conservation of canonical angular momentum makes the beam rotate around the magnetic axis and then propagate into a drift region containing plasma.

Experiments on the interaction between the rotating electron beam with the magnetically trapped plasma showed effects like excitation of a cross-field return current layer by the exiting beam, generation of magnetosonic waves by the return current layer and plasma heating by magnetosonic waves [16-18]. The device was also used to study the self-field effects and the effects of charge neutralization on the dynamics of intense beam propagation through non-adiabatic cusp fields [19]. The experiment was later modified for creating compact toroids by field reversal by the return currents excited by a rotating electron beam [20]. To get a higher energy, longer duration beam, the Marx generator was fired directly into the diode. The return current was high enough to reverse the original mirror magnetic field. This results in the formation of a field-reversed configuration commonly known as a compact torus.

\section{Toroidal Plasma Experiments in BETA}

We also set up an experiment to study what happens when a high current beam is injected into a toroidal system pre-filled with plasma [21]. Thus was built the first toroidal device in IPR, supported by a grant from the National Science Foundation, US and collaboration from Prof. Charles Wharton from the Cornell University, in which experiments on injection and stacking of high current electron beams produced by a graphite cathode driven by a pulse forming line charged by a Tesla transformer were done.

The toroidal plasma device evolved into BETA, an acronym for basic experiments in toroidal assembly. The device consists of a $45 \mathrm{~cm}$ major radius and $15 \mathrm{~cm}$ minor radius torus, formed by joining four SS quadrants. 16 picture frame coils around the torus produced a toroidal magnetic field of $0.1 \mathrm{~T}$ and $1.2 \mathrm{sec}$ duration. Energetic electrons from a hot tungsten filament produce plasma. A conducting grounded limiter of $9 \mathrm{~cm}$ radius short-circuits the charge separation electric field formed by the magnetic field curvature provides equilibrium.

Studies on plasma formation with the thermionic electron source established that discharge current depends upon the toroidal magnetic field. The discharge current saturates at a toroidal magnetic field that is much less than the self-magnetic field of the filament. Particle trajectories in the vicinity of the filament with varying velocity components at different spatial locations and magnetic fields show that escape or trapping of electrons emitted by the filament depends strongly on where the electron originated and its velocity [22]. Consequently, escaping electrons responsible for ionization are inhomogeneously emitted. The degree of inhomogeneity is reduced as the magnetic field increases, leading to saturation.

Low beta plasma embedded in a pure toroidal magnetic field is susceptible to different types of flute type instabilities [23-24] with frequency below the ion gyro frequency, namely the velocity- shear instability driven by non-uniform electric field; the Rayleigh-Taylor (R-T) instability arising from an unfavorable curvature in the magnetic field with 
density gradient antiparallel to it; and the gradient-drift instability that arises due to relative drift between the electrons and ions. Coherent low-frequency fluctuations are excited spontaneously at $0.02 \mathrm{~T}$. The low-frequency mode is identified as due to curvature-induced R-T instability favored by velocity shear.

BETA has also been used for understanding the poloidal plasma rotation induced by electrode biasing and its effect on fluctuations and confinement [25]. Poloidal plasma rotation and, concomitantly, significant reduction in the fluctuation level are observed. The radial profiles of poloidal flows and fluctuations are measured and compared. The dependence of the poloidal plasma rotation on the bias voltage and the toroidal magnetic field was investigated. It is found that the large poloidal plasma rotation gives rise to a transition of the plasma state from a turbulent to a coherent mode.

\section{Non-neutral Plasma Experiments}

Non-neutral plasmas are excellent experimental manifestations of 2-dimensional vortices in an inviscid fluid; surface perturbations of the electron column such as the diocotron mode resemble surface ripples in a fluid. Malmberg pioneered studies on linear non-neural plasma columns. A few toroidal experiments in large aspect ratio devices were application-driven to form deep potential wells for ion acceleration. The small aspect ratio limit was a virgin territory and we exploited this opportunity to do novel experiments. With the process of magnetic induction charging in which inward moving magnetic flux carried electrons introduced at the boundary, we produced an electron cloud, which demonstrated the inward-shifted equilibrium and other features of electron plasmas in toroidal traps. The first paper on the equilibrium features came out in 1992, which incidentally, was the first Physical Review Letters paper in experimental plasma physics from India [26]. Interesting complementarity between charged non-neutral plasmas and current carrying neutral plasmas, like the capacitive effects replacing inductive effects etc. are discussed in a review paper [27].

We invented a method of plasma formation based on the modification of the vertical drifts into closed diocotron drift trajectories by a combination of self-consistent space charge electric field and an externally applied radial electric field. The cross-field flow develops into a non-neutral cloud by the following mechanism. When it is radially located close to the inner wall, it induces a net image charge on the wall and a resultant outward electric field. The guiding centre of the particles will be slowed down due to the interaction of the image electric field with the particles. This increases the density, increasing the image electric field, further slowing down the particles, amplifying the density clump initially created by the retardation of the flow by the image electric field [28].

The turbulent birth of toroidal non-neutral plasmas by cross-field transport was a fundamental difference from the near-equilibrium placement in the Malmberg trap. We speculated that the plasma formed by injection parallel to the B-field would be more quiescent. However, to do this in a torus, the filaments would have to be placed inside the drift space; the torus would no longer be closed. Thus was born The SMARTEX-C device [29]. A single circular tungsten filament loop, placed on a poloidal cross section, emits thermionic electrons when heated. A negatively biased grid placed in front of the filament is pulsed positive to extract electrons parallel to the minor axis. Another grid collector placed behind the filament in the poloidal cross section, is biased negative. As the toroidal magnetic field, established by pulsing a current through a multi-turn coil, reaches its flat top, the injector grid is pulsed positive with respect to filament to extract electrons along the field lines. Thereafter the grid reverts to negative bias, stopping further fuelling. The injected electrons are now trapped toroidally between the negatively biased injector grid and collector grid.

The successful confinement in the small aspect-ratio limit has led to several interesting observations: the evolution of the confined plasma is marked by large amplitude electrostatic wave activity. Coherent, periodic, double peak oscillations result from low-frequency ExB motion of a toroidal vortex that leans closely against the inner wall. As many as 16 highly phase-coherent harmonics with dominant power in $\mathrm{m}=2$ suggest that the mode is not a center-of-charge motion, but a strong coupling of modes resulting in a novel nonlinear state. The absence of any power-law tail suggests absence of turbulence. The frequency, around $100 \mathrm{kHz}$ at 200 Gauss reduces as the mode evolves, but later increases as the mode dies.

\section{Large Volume Plasma Device}

Fundamental processes in naturally occurring plasma will result in high energy release, generation of powerful electromagnetic radiation, and acceleration of energetic particles. These processes are highly complex due to multiplicity of interactions between fields and flows in the plasma.

Direct probing of space plasma precludes control over the processes. A laboratory device with minimum boundary effects where controlled experiments can be carried out would be very useful. The device must be large enough to minimize boundary effects from perturbing the phenomena studied and accommodate many electromagnetic wavelengths along and across the magnetic field. Secondly, as the magnetic field provides directionality to the ionizing electrons, providing a uniform, large area source of primary electrons becomes difficult, resulting in non-uniformity of plasma parameters and generation of large noise, especially during plasma production. The multiplicity of parameters that need to be measured with adequate temporal and spatial resolution is another concern. 
The Large Volume Plasma Device (LVPD) meets many of these requirements [30]. A $60 \mathrm{~cm}$ by $60 \mathrm{~cm}$ multi filamentary cathode, produces large volume, quiescent plasma of density $1012 / \mathrm{cm} 3$, with $5 \%$ uniformity over $1 \mathrm{~m}$ axially and radially. The influence of energetic electrons on the phenomena under study can be eliminated by doing experiments in the afterglow of highly reproducible pulsed discharges. Measurement over a large volume with high spatial and temporal resolution, acquisition, and storage of voluminous data form other challenges.

LVPD has been used for many basic studies. Whistler waves have been excited by an antenna system consisting of an array of phase compensated insulated wires, separated by a skin depth. Observations show that, instead of forming a current sheet, whistler waves are excited by each current element. The resulting wave field at any spatial point is a linear superposition of waves excited by individual current elements. The results also indicate that the magnetic field variations of the whistler wave in the far-field region are independent of the current source configuration, whereas in the near-field region, they depend on the antenna configuration.

In the case of wave excitation by a conduction current method, it is the extent of the return current system, and not the extent of the electrode, that determines the perpendicular wave vector of the excited whistler wave. Experiments related to excitation of hollow return current system have established that the wave excitation does not originate from the surface of the electrode, but from a distance 5 skin depth inside the hollow current system. This probably indicates that the source region of the wave excitation field has finite depth.

The most recent observation is that of electron temperature gradient (ETG) driven turbulence in the laboratory plasma [31]. Removal of unutilized primary ionizing and non-thermal electrons from uniform density plasma and imposition and control of gradient in electron temperature are all achieved by placing a large $(2 \mathrm{~m}$, diameter $)$ magnetic Electron Energy Filter (EEF) in the middle of the device. In the dressed plasma, the observed ETG turbulence in lower hybrid range of frequencies $1-80 \mathrm{kHz}$ is characterized by a broadband with a power law.

\section{Helicon Waves in Torus}

Bounded whistlers are efficient plasma generators in cylindrical tubes. Breakdown and discharge studies in toroidal systems were initiated in 1990s [32]. After the breakdown, the discharge is sustained by toroidal bounded whistlers. With pulsed experiments, the time evolution of the discharge could be studied in four distinct phases of RF breakdown, steady state attainment, decay and afterglow. In the steady state, average electron density of $10^{12}$ per cc and average electron temperature of $20 \mathrm{eV}$ are obtained. Experiments revealed the roles of toroidal mode structure, background effects and time evolution of the electron distribution function in the breakdown mechanism.

Helicon wave experiments in a small aspect ratio torus followed [33]. Owing to the strong poloidal asymmetry in the wave magnetic field structures, a non-resonant current is driven in plasma by the dynamo electric field, which arises due to helicity injection by helicon waves. A study of the parametric dependence of plasma current driven in the high frequency electron magneto hydrodynamic regime, along with numerical estimations of non-resonant components, was done. The measured magnitude of plasma current is in good agreement with estimated values. Numerical estimation, using experimentally measured variations of wave magnetic field components, clearly delineates the plasma current due to wave-induced helicity from other possible resonant or non-resonant sources in the given parameter regime.

\section{Dusty Plasma Experiments}

The dust acoustic wave (DAW) is a low frequency collective mode of the dusty plasma that has been extensively studied in the past few years. DAW is a modified version of the ion acoustic mode of ordinary plasma, where the inertia is now provided by the massive and highly charged dust grains and the thermal pressure sustaining the wave comes from both the plasma electrons and ions. Due to the large inertia of the dust grains, their sluggish response to an electric field results in very slow oscillations, typically in the range of a few hertz. Experimental studies have focused on the nonlinear state of a spontaneously excited dust acoustic wave in a strongly coupled dusty plasma [34]. The nonlinear state is obtained by controlled decrease of the background neutral pressure which concomitantly decreases the wave damping from dust neutral collision processes.

The DAW is seen to evolve from an initial coherent state to an increasingly turbulent state through the excitation of higher harmonic components [35]. A nonlinear saturation is based on a process whereby the energy of the long wavelength fundamental DAW is nonlinearly transferred by mode coupling to viscosity damped shorter wavelength harmonics. The viscosity coefficient used in the model is a function of the Coulomb coupling parameter. Its value is somewhat approximate due to uncertainties in the experimental evaluation of the Coulomb parameter. The principal difficulty is in the estimation of the dust temperature. Calculation of the dust temperature assumes that the dust grains are in thermal equilibrium with the neutral gas molecules.

\section{ADITYA Tokamak}

In the early 80 s, a proposal to the Department of Science \& Technology, Government of India to build and operate a medium sized tokamak device was approved as the Plasma Physics Programme in 1982. By 1984 the activities moved into the campus at Bhat village in the outskirts of Ahmedabad city. The PPP evolved into the autonomous 
Institute for Plasma Research under the Department of Science and Technology in 1986.

ADITYA, the first Indian tokamak (Fig.8), conceived, designed, largely indigenously fabricated and erected at the IPR, is a medium size low field device [36]. Commissioned in 1989, it has plasma of circular cross-section with major radius $0.75 \mathrm{~m}$ and minor radius of $0.25 \mathrm{~m}$. A toroidal field of up to $1.5 \mathrm{~T}$ can be produced at plasma center with the help of 20 toroidal field coils spaced symmetrically around a toroidal vacuum vessel. The chief scientific objectives of ADITYA are (i) investigation and control of edge phenomena; (ii) investigation of density and current limits of a tokamak with special emphasis on interesting phenomena like MARFES, detached plasma, disruptive instabilities and their control; (iii) study of novel regimes of operation such as those with currents dominantly in energetic current carriers etc.

The Ohmic transformer for ADITYA consists of a central solenoid, which stores the required flux and three additional pairs of compensating coils in order to minimize the field within the plasma region. The solenoid produces a peak field of 3.2 Tesla within the bore to store a flux of 0.6 Volt-sec at peak current of $20 \mathrm{kA}$. The vertical field for ADITYA is generated by means of two pairs of the vertical field coils designed to carry $10 \mathrm{kA}$ peak current.

The vacuum vessel of ADITYA is a torus of major radius $75 \mathrm{~cm}$ with a square cross-section of side $60 \mathrm{~cm}$, a volume of $\sim 16 \mathrm{~m}^{3}$ and total surface area of $\sim 75 \mathrm{~m}^{2}$. The vessel, designed for ultra-high vacuum (UHV), is made out of SS $304 \mathrm{~L}$ material and assembled in four quadrants. The vacuum vessel has been subjected to various wall treatment procedures in order to be compatible for UHV $\sim 10^{-8}$ torr. A combination of Glow Discharge Cleaning and low temperature Pulsed Discharge Cleaning is used for keeping the surface clean. The vacuum vessel is pumped by two turbo-molecular pumps each having a pumping capacity of 2000 liters per second for air. In addition, during discharge cleaning, two cryopumps, each having a pumping capacity of 10,000 liters/sec for water vapour and condensable hydrocarbon, are also used.

Aditya has a very sophisticated pulsed power system for a machine of this size. It is based on a set of line commutated converters connected to the Gujarat Electricity grid through a 50 MVA substation and providing shaped power pulses for the magnetic field coils. Pulsed power ( $\sim 50 \mathrm{MW}$ per pulse) required to energize the magnetic field coils of ADITYA is drawn from the electricity grid. Controlled rectifiers cum wave shaping circuits convert the mains power into pulses required for the operation of the tokamak.

A large number of key diagnostics e.g. microwave interferometer, Laser Thomson scattering, Visible and UV spectroscopy, soft X Ray imaging, ECE radiometer, Langmuir and magnetic probes have been developed and deployed on ADITYA. The device operates under full control of automated data acquisition and control system.

In tokamaks, edge and core plasma are integrated and phenomena in edge influence core transport profoundly.
Experiments in ADITYA have been dedicated to edge turbulence studies. The intermittency in tokamak edge turbulence was discovered in ADITYA. The probability distribution functions of the density and potential fluctuation were found to be non-Gaussian (figure 5). This was followed up by first observation of coherent structures in conditional statistical analysis of the fluctuations (figure 6). The results indicated the particle transport in tokamaks is not the steady ooze but comes out in bursts. Other important experimental results include scale dependence of the fluctuation dependent particle transport, \& Levy's statistics of edge turbulence.

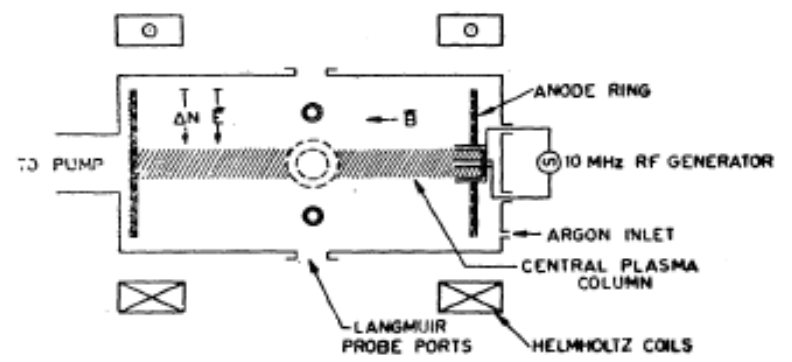

Figure 1. Schematic diagram of the Electrojet Simulation Experiment

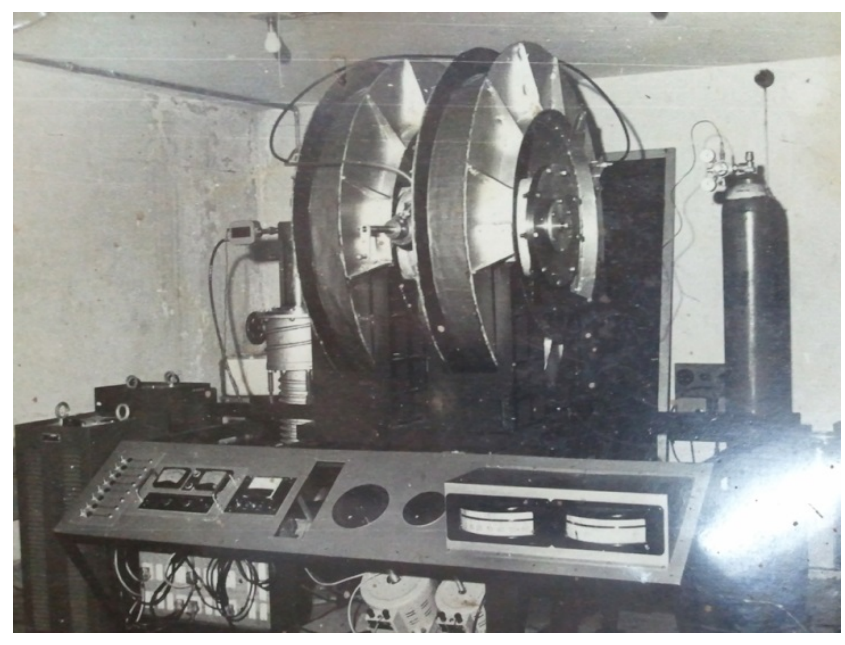

Figure 2. Photograph of the Device in which the Electrojet Instabilities were simulated. The Huge magnet coils were wound using resistive copper wire

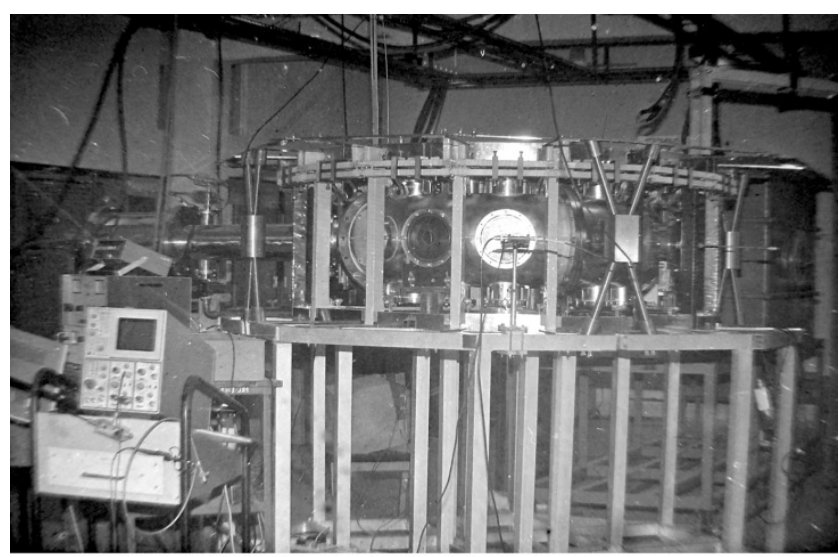

Figure 3. The first toroidal plasma device built at the Institute for Plasma Research in which an intense electron beam was injected to form an electron beam ring 


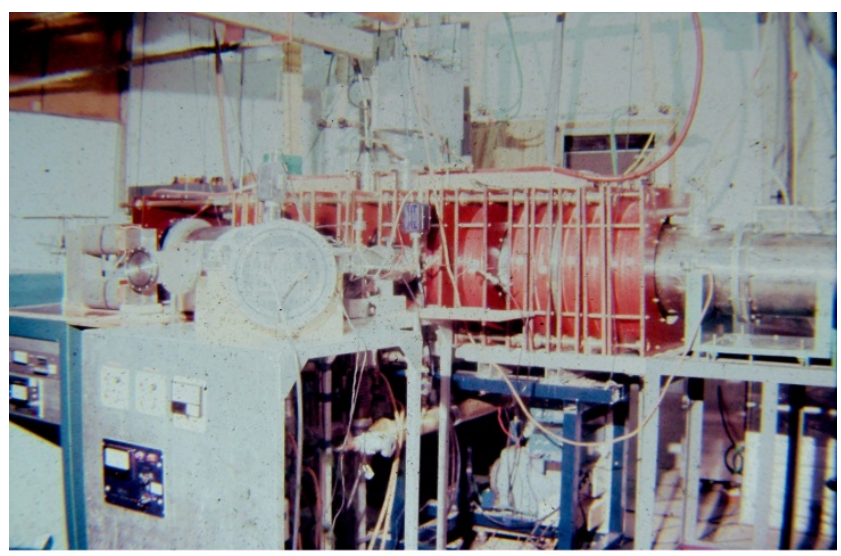

Figure 4. The REB-Plasma Interaction experiment which was used to study plasma heating by return currents

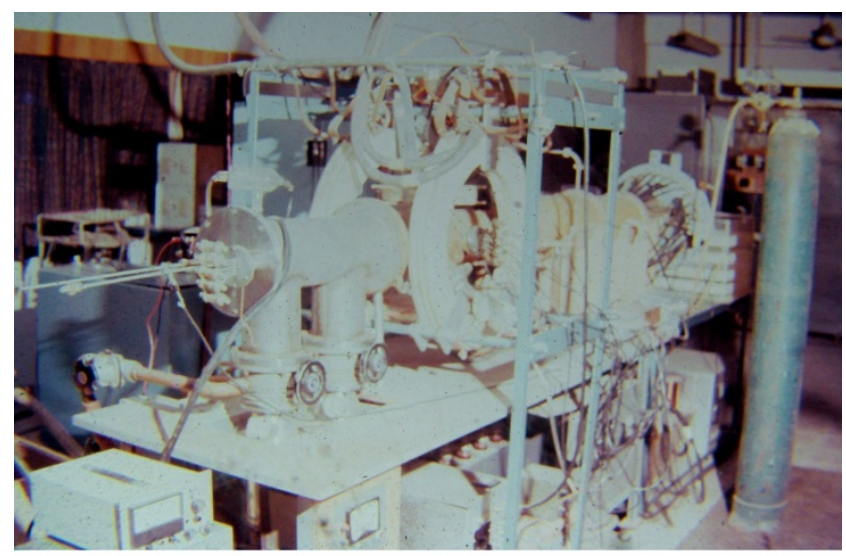

Figure 5. Plasma-Neutral gas interaction experiment to study the Alfven Critical Velocity Effect
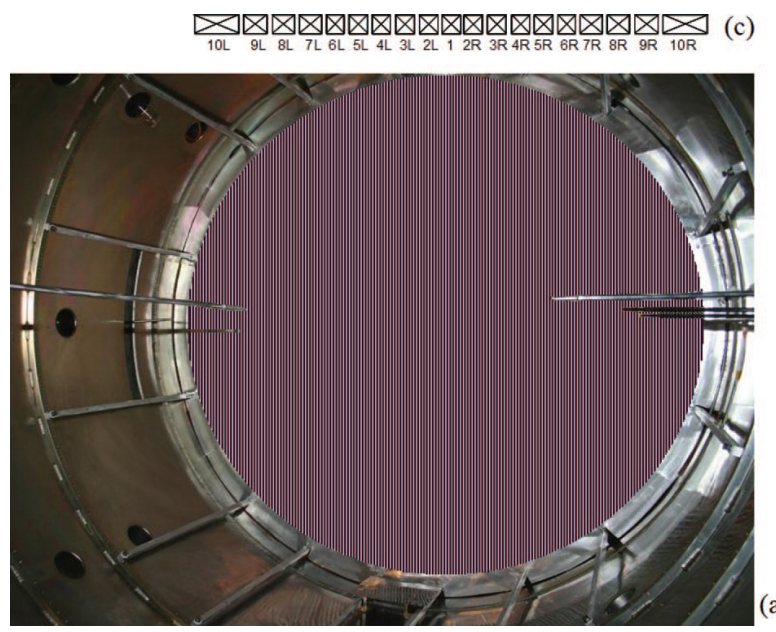

\section{(c)}

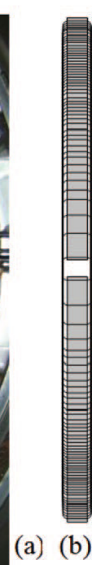

Figure 6. (a) Photograph of the installed EEF in LVPD, (b) the top view showing the extent of respective coils and (c) the side view of its cross-section. The design allows $82 \%$ physical transparency between plasma of source and target region.

ADITYA has been upgraded recently to obtain better parameters and performance. The vacuum system has been improved by improving the surface cleaning facilities. To increase the plasma energy content during the discharge, auxiliary heating systems have been integrated. A $20-40$ MHz, 200 KW Ion Cyclotron Resonance Heating (ICRH) system has been integrated to ADITYA vacuum vessel. A 28 $\mathrm{GHz}, 200 \mathrm{KW}$ gyrotron based electron cyclotron resonance heating $(\mathrm{ECRH})$ system has been successfully commissioned on ADITYA tokamak. ADITYA is regularly being operated with the transformer-converter power system with $\sim 100$ msec $80-100 \mathrm{kA}$ plasma discharges at toroidal field of 8.0 $\mathrm{kG}$.

\section{The Steady State Superconducting Tokamak}

The mid-1990s saw a major spurt in our activity when a proposal to build a state-of-the-art superconducting steady-state tokamak (SST) received official support. At this point, the institute also changed its departmental affiliation from Department of Science \& Technology to the Department of Atomic Energy.

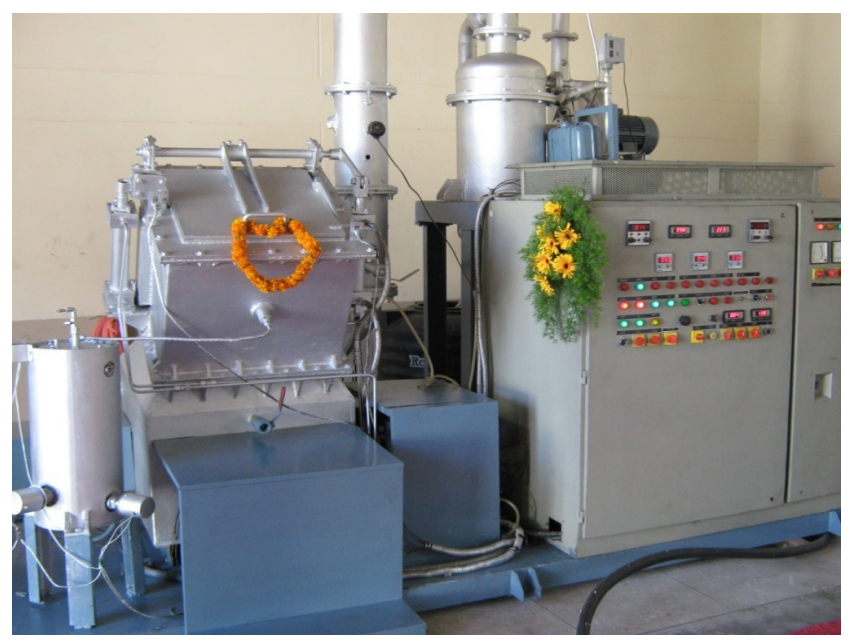

Figure 7. The commercialized system developed at the Facilitation Centre for Industrial Technologies for the destruction of medical waste with Plasma Pyrolysis.

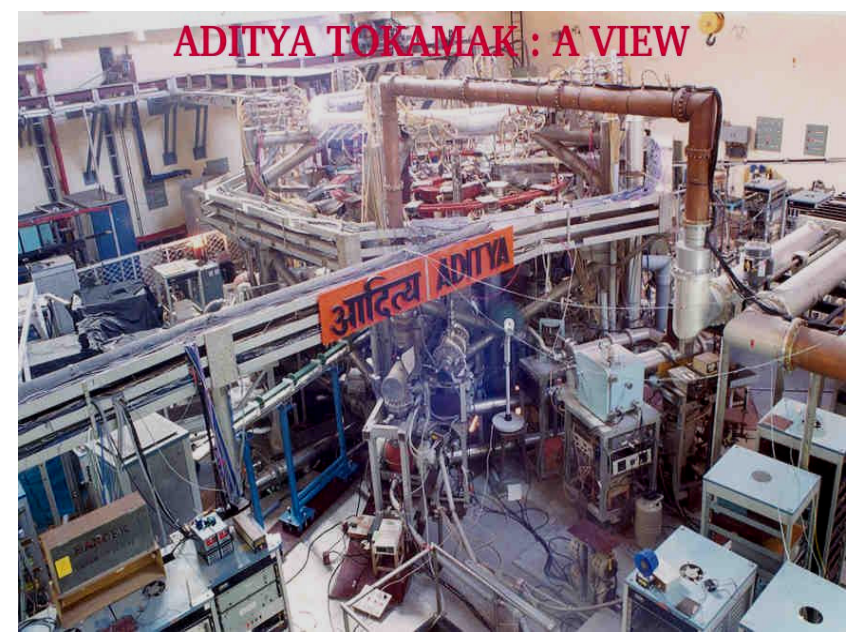

Figure 8. ADITYA, the first Indian tokamak commissioned in 1989

The SST-1 tokamak (Fig.9) is a current-generation 
tokamak, which has started operation [37]. It is fitted with superconducting magnets and is designed to produce a long-pulsed plasma lasting for nearly, 1000-seconds. The plasma will be sustained by RF-assisted current drive and heated with auxiliary RF power as well as energetic neutral beams.

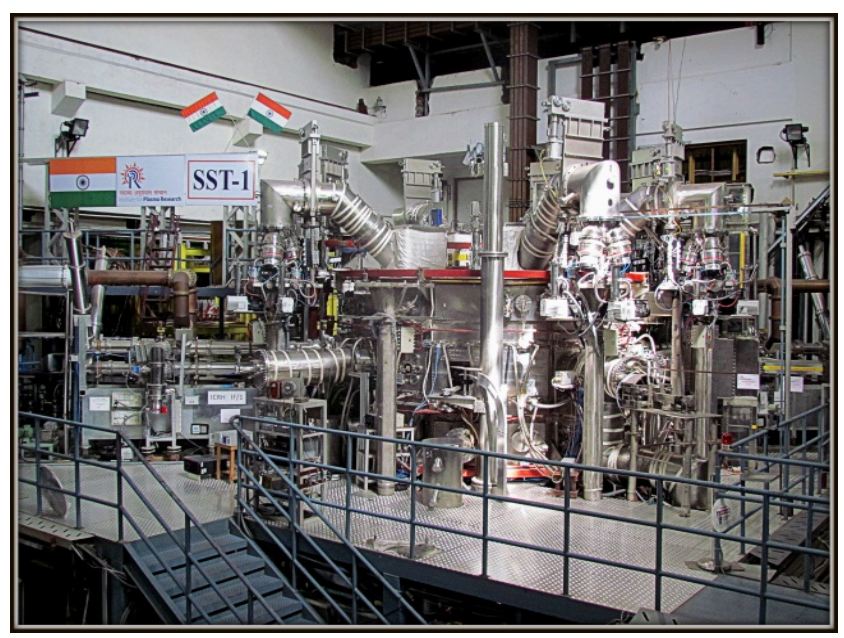

Figure 9. Super Conducting Steady State Tokamak built and commissioned indigenously

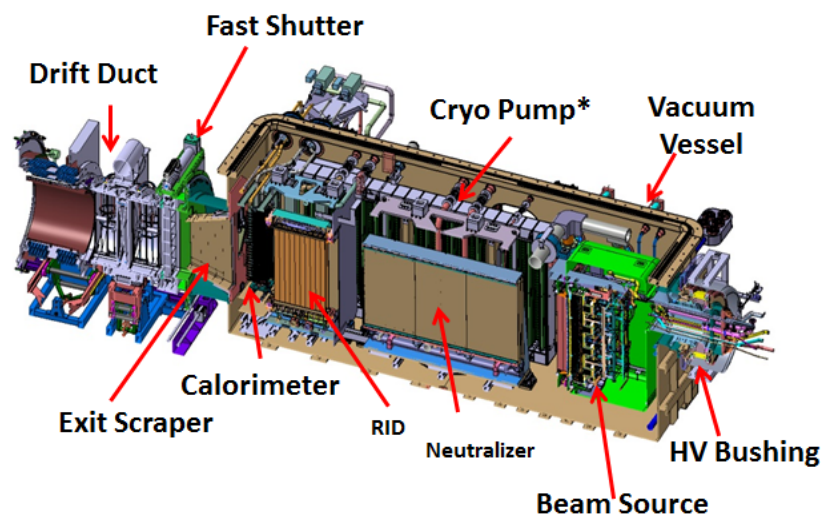

Figure 10. A schematic figure of the $100 \mathrm{KeV}$ Diagnostic Neutral Beam which is part of the Indian Procurement Package to ITER

SST-1 plasma will provide an opportunity to investigate several front-line physics issues related to particle and energy confinement as well as to stability and control of 'steady-state' plasmas.

Steady State Superconducting Tokamak (SST-1) has made significant progresses both on enhancing the parameter space of operations as well as up-gradations of its core sub-systems. SST-1 completed the $1^{\text {st }}$ phase of up-gradation with successful installation and integration of all its First Wall components on May 29, 2015. The First Wall of SST-1 comprises of $\sim 4500$ high heat flux compatible graphite tiles being assembled and installed on $130 \mathrm{CuCrZr}$ heat sink back plates engraved with $\sim 4 \mathrm{~km}$ of leak tight baking and cooling channels in five major sub groups equipped with $\sim 400$ sensors and weighing $\sim 6000 \mathrm{~kg}$ in total in thirteen isolated galvanic and six isolated hydraulic circuits. The phase-1 up-gradation spectrum also included addition of Supersonic Molecular Beam Injection (SMBI) both on the in-board and out-board side, installation of fast reciprocating probes, adding some edge plasma probe diagnostics in the SOL (Scrape Off Layer) region, installation and integration of segmented and up-down symmetric radial coils aiding/controlling plasma rotations, introduction of plasma position feedback and density controls etc. Post phase-I up-gradation, a robust First Wall baking system assisted with hot Nitrogen and capable of baking the plasma facing components in excess of $300 \mathrm{C}$ over long duration has been established. In order to make the SST-1 Poloidal Field (PF) Magnets superconducting, an elaborate modification in the 'integrated flow distribution system' to various similar groups of hydraulic paths of the superconducting magnets system have also been carried out. After initial engineering validations of the up-graded SST-1 that included establishment of $10^{-8}$ mbar order vacuum inside the vacuum vessel and helium based 'glow discharge cleaning; initial plasma experiments in up-graded SST-1 have begun since Aug 2016. The first experiments in SST-1 have revealed interesting aspects on (a) the 'eddy currents in the First Wall support structures' influencing the 'magnetic Null evolution dynamics' (b) the subsequent plasma start-up characteristics after the ECH pre-ionization (c) the influence of the first walls on the 'field errors' and the resulting locked modes (d) the magnetic index influencing the evolution of the equilibrium of the plasma column (e) low density supra-thermal electron induced discharges and normal ohmic discharges etc.

Presently; repeatable Ohmic discharges regimes in SST-1 having plasma currents in excess of $100 \mathrm{KA}\left(\mathrm{q}_{\mathrm{a}} \sim 2.8, \mathrm{~B}_{\mathrm{T}}=1.5\right.$ $\mathrm{T})$ up to a maximum of $112.5 \mathrm{KA}$ with a current ramp rates $1.2 \mathrm{MA} / \mathrm{s}$ over a duration of $\sim 500 \mathrm{~ms}$ with line averaged densities $\sim 0.8-1.0 \times 10^{19}$ and temperatures $\sim 200-300 \mathrm{eV}$ with copious MHD signatures have been experimentally established. Further elongation of the plasma duration up to one second or more with position and density feedback as well as coupling of Lower Hybrid waves are currently being persuaded in SST-1. Experimental thrust area will now be on the edge plasma turbulences, particle and energy transports, MHD activities and disruptions.

\section{Plasmas for Profit}

After commissioning ADITYA, using plasmas for near-term industrial applications appeared to be very timely [38]. The rich experimental knowledge base created over the years in producing and manipulating plasmas to support basic research and fusion both at PRL and IPR was the capital.

Plasma processing was surging internationally with the realization that the fourth state of matter offers unique opportunities in high energy density material processing with high value addition. Industrial plasma applications are 
mediated by properties like high chemical reactivity, microscopic electric fields, sheaths, radiation and particle flux. Plasma based manufacturing integrates the plasma-material interaction phenomena with the manufacturing process. The technology adds value to conventional materials and makes new types of materials and processing techniques possible. The characteristics of both the equilibrium and non-equilibrium plasmas can be exploited for commercial uses.

Comparable stature of international and indigenous capability in this field was a rare opportunity for leadership in an emerging field.

There were no pre-existing business models of similar activity in basic research organizations in India. The business plan evolved and matured along with our learning curve. The plan for converting the knowledge base to commercially viable technologies conceived technology as the product and the industry as the market.

The programme had to be entrepreneur-driven to make it agile and responsive to rapid changes and focused on a few thrust areas where immediate impact would be possible. Financial self-reliance was a goal from the beginning.

The industrial plasma activity, started in the early nineties with plasma nitriding flowered into a large variety of applications, using both thermal and nonthermal plasmas. New recruits brought much-needed expertise in fields such as chemistry, material sciences and metallurgy.

In 1997 we set up the Facilitation Centre for Industrial Plasma Technologies to consolidate all activities related to technology development, demonstration, incubation and commercialization.

With time, Plasma processing has transcended conventional material processing applications into waste destruction, environmental remediation, water purification, flue gas treatment etc. It is emerging as an enabling tool with a wide spectrum of applications relevant to the modern industrial society. At FCIPT, on the basis of an assessment of the industrial sectors likely to benefit from the new technological inputs, a few selected areas were focused upon. These included surface engineering, mineral processing, advanced ceramics, photovoltaic devices, environmental remediation, thin film deposition, and biomedical applications. Three major technologies that FCIPT has developed indigenously are Plasma Nitriding, Plasma Pyrolysis, and Plasma Enhanced Chemical Vapour Deposition (PECVD).

FCIPT is a path breaker in India in converting physics based research into commercially valuable devices and processes (Fig.8). With the new thrust in manufacturing and with the growth of strategic programmes, this activity will be timely and rewarding.

\section{ITER}

In 2004, India's possible entry into ITER was being discussed among the science planners. Collaboration in fusion research under ITER was mooted at the Indo-E.U. summit in November 2004. The thaw in Indo-US relations also helped.

India's formal request to join ITER was considered by the ITER negotiating committee at its September 2004 meeting at Cadarache. An ITER fact-finding mission visited India in October 2005. The mission visited the IPR and other centres to appraise itself of the Indian fusion research programme as well as industrial capabilities relevant to ITER.

India was admitted as a full partner at the ITER meeting at Jeju Island in December 2005. Agreement for Accession to ITER was signed in 2006. India's participation in ITER will be in terms of supply and manufacturing of some components and sub-systems of ITER (Fig.10). To deliver this, an entity called ITER-India has been formed [39].

\section{National Fusion Programme}

India will learn $10 \%$ of ITER through the supply of hardware. The 'Balance of ITER' will have to be learnt through an indigenous programme covering core fusion technologies like fusion materials, fusion neutronics, RF and microwave power systems, power engineering, data acquisition and control systems, plasma diagnostics, robotics, super conducting magnets and cryogenic technology etc.

The National Fusion Programme (NFP) is aimed at acquiring indigenous competence in all these fields [40]. The programme is multidisciplinary, multi-institutional in character. In broad terms, we must generate a community of fusion physicists, set up programmes for 'Balance of ITER' technologies, partner with industries and develop human resources.

NFP programme is designed to extend and complement the IPR efforts in all areas of fusion science and technology. Internal fine-tuning of the proposals and having IPR personnel to act as co-ordinators ensure this.

Up to now we have funded more than 100 projects with an outlay of 30 Crores. The project is managed by the Plasma and Fusion Research Committee (PFRC) of the Board of Research in Nuclear Sciences (BRNS) of the Department of Atomic Energy.

\section{Conclusions}

From a modest start in the early 1970s, India has made great, largely self-reliant strides in the field of experimental plasma physics. Capacity building in techniques relevant to plasma production, manipulation and control, creation of magnetic field and geometrical configurations and development and deployment of diagnostics to enable understanding of fundamental processes in plasmas has been truly remarkable. It is through these experiments that the human resource and technology development essential to sustain India's ambitious foray into magnetic confinement 
fusion and industrial and strategic plasma applications has taken place.

Where will Indian Plasma Physics go from here? The Indian Roadmap for fusion mentions the construction of next generation SST by 2030 and a DEMO by 2040-45. Lessons from ITER will be essential to render more clarity to this. The broad-basing of research in Universities and National Institutions through NFP will help push research in fusion and applications and contribute to human resource. Technology, like superconducting magnets and high voltage power supplies developed for Fusion will permeate to non-Fusion applications. Plasma Science has a natural frontier with most branches of modern physics and technologies and therefore, basic plasma research, critical for human resource development, will continue strongly at IPR and other universities and major institutions. Societal contributions, a strong component of the Indian programme will continue to attract new ideas and investment. Similarly, strategic areas and space research will continue to grow to dynamically relevant levels.

\section{Acknowledgements}

The work reported above has been extracted from the contributions of a large number of people; too numerous to be named. I am aware of the fact that I have left out references to a number of organizations that were also part of this journey. The excellent work at BARC, CAT, SINP, CPP and IITs were not covered.

\section{REFERENCES}

[1] P K Kaw and A Sen in India in the World of Physics: Then and Now, Chapter 14, Pearson Education India 2009

[2] P I John and Y C Saxena: Observation of Farley-Buneman Instability in laboratory plasmas, Geophys. Res. Letters, $2,251,1975$

[3] Y C Saxena and P I John: Observation of the cross-field instability in laboratory plasma, Geophys. Res. Lett. 2,492, 1975

[4] P I John and Y C Saxena: Dispersion and spectral characteristics of cross-field instability in collisional magnetoplasmas, Pramana, 8,123, 1977.

[5] D Bora, P I John, Y C Saxena and R K Varma: Multiple life times in the nonadiabatic leakage of charged particles from a magnetic trap, Plasma Physics, 22,653, 1980

[6] D Bora, P I John, Y C Saxena and R K Varma: Behaviour of charged particles confined in a magnetic mirror, Phys. Fluids, 25, 2284, 1982

[7] P I John and Y C Saxena: Propagation of ion acoustic solitons at plasma density gradients, Phys.Lett, 56A, 385, 1976
[8] P I John and Y C Saxena and R P Dahiya: Ion acoustic solitons in the presence of strong radiofrequency fields, Phys.Lett. 60A,119, 1977

[9] R P Dahiya, P I John and Y C Saxena: Reflection of ion acoustic solitons at a negatively biased grid, Phys.Lett. 65A, 323,1978

[10] S Mukherjee and P I John: Dynamics of a collisional ion sheath, Pramana, 44, 55, 1995

[11] S Mukherjee, K Avinash and P I John: Dynamic sheath expansion and ion current in transient ion sheath experiments, Pramana 44, 263, 1995

[12] S.K. Mattoo and N Venkataramani: On the threshold velocity in the interaction between a magnetized plasma and neutral gas, 76A, Physics Letters 1980

[13] N Venkataramani and S K Mattoo: Plasma retardation in Alfven critical velocity phenomenon, Physics Letters 79A, 1980

[14] N Venkataramani and S K Mattoo: On plasma-neutral gas interaction Pramana, 15, 117-136, 1980

[15] K K Jain and P I John: Gigawatt power electron beam generator: Proc. Indian Acad Science, 4, 75, 1981

[16] P I John: Plasma Heating by rotating relativistic electron beams: Physica Sripta, T2:2, 298, 1982

[17] K K Jain and P I John: Rotating relativistic electron beam plasma interaction and formation of a field reversed configuration: Pramana, 23, 1, 1984

[18] K K Jain and P I John: Interaction of a rotating relativistic electron beam with a plasma: Plasma Physics and Controlled Fusion, 26, 891, 1984

[19] M K Vijayashankar and P I John: Effects of self-fields on the dynamics of relativistic electron beams in a cusp magnetic field: Plasma Physics and Controlled Fusion, 31, 813, 1989

[20] K K Jain and P I John: Formation of a compact torus by a rotating relativistic electron beam: Phys. Fluids, 29, 3445, 1986

[21] D Chenna Reddy and P I John: Formation of an REB ring in a toroidal device: Proc. International Conference on Plasma Physics, New Delhi, Vol. III 977, 1989

[22] R Kaur and S K Mattoo Inhomogeneous electron emission from a hot filament in a toroidal magnetic field, Plasma Sources Science and Technology, Volume 18, Number 1 0150032008

[23] Territorial characteristics of low frequency electrostatic fluctuations in a simple magnetized torus, R. Kaur, A. K. Singh, R. Singh, A. Sarada Sree, and S. K. Mattoo Physics of Plasmas 18, 012109 (2011)

[24] G Prasad, D Bora, Y C Saxena and G C Sethia, ,Ä Study of low-frequency flute-type coherent fluctuations in a toroidal plasma, Plasma Phys. Control. Fusion 37, 387, 1995

[25] K.K. Jain Poloidal plasma rotation and its effect on fluctuations in a toroidal plasma, Nuclear Fusion, Volume 36, Number 12

[26] P Zaveri, P I John, K Avinash and P K Kaw: A low Aspect 
Ratio Toroidal Non-Neutral Plasma Physical Review Letters, $68,3295,1992$

[27] P I John: Physics of Toroidal Electron Clouds, Plasma Phys and Contr Fusion 34, 2053, 1992

[28] S S Khirwadkar, P I John, K Avinash, A K Agarwal and P K Kaw: Steady State Formation of a Toroidal Electron Cloud Physical Review Letters, 71, 4334, 1993

[29] S Pahari, H S Ramachandran, P I John: Electron plasmas: Confinement and mode structure in a small aspect ratio toroidal experiment, Phys of Plasmas, 13, 092111, 2006

[30] S. K. Mattoo, V. P. Anitha, L. M. Awasthi, G. Ravi, and LVPD Team, A large volume plasma device, Review of Scientific Instruments, Vol 72, 1.1405793, 2001

[31] S K Singh, L M Awasthi, S K Mattoo, P K Srivastava, R Singh and P K Kaw, Investigations on ETG turbulence in finite beta plasmas of LVPD, Plasma Physics and Controlled Fusion, Vol 54, 124015, 2012

[32] S K P Tripathi, D Bora and M Mishra, RF breakdown by toroidal helicons, Pramana 56, 551, 2001
[33] Manash Kr Paul and D Bora: Wave-induced helicity current drive by helicon waves Physics of Plasmas 14, 082507, 2007

[34] J Pramanik, B M Veeresha, G Prasad, A Sen, P K Kaw: Experimental observation of dust-acoustic wave turbulence, Physics Letters A 312, 84, 2003

[35] P Bandyopadhyay, G Prasad, A Sen, P K Kaw: Experimental study of nonlinear dust acoustic solitary waves in a dusty plasma, Phys Rev Lett. 101, 065006, 2008

[36] S B Bhatt and the ADITYA team, Aditya: The first Indian Tokamak, Indian J. Pure and Applied Physics. 27, 710, 1989

[37] S. Pradhan and the SST-1 Team, The first experiments in SST-1, Nuclear Fusion, Volume 55, 10040092015

[38] P I John, Commercialization of plasma-assisted technologies: the Indian experience, Physica Scripta, Volume 2002, 86, 2002

[39] ITER-India website: https://www.iter-india.org/

[40] The National Fusion Programme website: www.ipr.res.in/NFP/ 